\title{
Canonical Treatments for the Two Bodies Problem with Varying Mass Taking into Consideration the Periastron Effect
}

\author{
M. I. El-Saftawy ${ }^{1,2}$, Amirah R. Algethami ${ }^{1}$ \\ ${ }^{1}$ Astronomy Department, Faculty of Science, King Abdu Aziz University, Jeddah, Saudi Arabia \\ ${ }^{2}$ Space Science Laboratory-NRIAG, Cairo, Egypt \\ Email: malsaftawy@kau.edu.sa
}

Received 16 January 2014; revised 10 February 2014; accepted 17 February 2014

Copyright $@ 2014$ by authors and Scientific Research Publishing Inc.

This work is licensed under the Creative Commons Attribution International License (CC BY). http://creativecommons.org/licenses/by/4.0/

(c) (i) Open Access

\begin{abstract}
In this work, the Hamiltonian of the two body problem with varying mass was developed in the extended phase space taking into consideration the periastron effects. The short period solution was obtained through constructing a second order canonical transformation using "Hori's" method developed by "Kamel". The elements of the transformation as well as the inverse transformation were obtained too. The final solution of the problem was derived using "Delva-Hanslmeier" method.
\end{abstract}

\section{Keywords}

Two Body with Varying Mass; Perturbation; Hori's Method

\section{Introduction}

The problem of the two bodies with varying mass has roots going back in the history since the middle of the 19th century. Since F. Mestschersky's initial study a great many researchers have dedicated much of their time to this problem, telling it became one of the classics of celestial mechanics, which has become known as the Gylden-Mestschersky.

An ample bibliography can be found in the published works of E. N. Polyakova [1] and C. Prieto [2]. The specific case which results in a slow isotropic mass loss has also been the focus of exhaustive studies carried out by researchers, for instance, J. D. Hadjidemetriou [3] [4], to name but a few. The vast majority of these, in search of the stellar application, have taken the so-called Eddington-Jeans law [5] [6], as a law of the variation of mass, 


$$
\dot{m}=-\alpha m^{n}
$$

where $\alpha$ and $n$ are real numbers, the first of them is positively proximate to zero and the second is varying between 1.4 and 4.4 .

Prieto [7] [8] intended to present an approximate analytic solution of the two-body problem with slowly decreasing mass which is obtained through the integration of the Hamilton equations using Deprit's method of perturbations. The solution, obtained through the Eddington-Jeans law, is put into practice in a specific case and compared with F. Mestschersky's exact equation; $n=2$; and with that which results from numerically integrating the equations.

In the framework of celestial mechanics, this problem has been exhaustively addressed by Docobo [9], Andrade [10], among others.

In 2002, M. Andrade [11] analyzed the dynamics of binary systems with time-dependent mass loss and periastron effect i.e., a supposed enhanced mass loss during periastron passage by means of analytical and numerical techniques.

M. Andrade and J. A. Docobo [12] studied the dynamics of binary systems with small parameter perturbation model, the time-dependence of the whole set of orbital elements, concluded, could be calculated over long timescales and even for high eccentricities. In these models, they studied the following time- and distance-dependent mass-loss law:

$$
\dot{\mu}\left(t ; r ; P_{\theta}\right)=\dot{\mu}(t)-\beta \frac{P_{\theta}}{r^{2}}
$$

where the first term represent time-dependent mass loss, and the last one introduces the periastron effect, where " $r$ " is the distance between the two components, $P_{\theta}$ is the total angular momentum and $\beta$ is another small parameter close to zero.

W. A. Rahoma et al. [13] were introduction paper concerned with the two-body problem with varying mass in case of isotropic mass loss from both components of the binary systems. The law of mass variation used gives rise to a perturbed Keplerian problem depending on two small parameters. The problem is treated analytically in the Hamiltonian frame-work and the equations of motion are integrated using the Lie series developed and applied, separately by Delva [14] and Hanslmeier [15]. A second order theory of the two bodies eject mass was also constructed, returning the terms of the rate of change of mass up to second order in the small parameters of the problem.

W. A. Rahoma et al. [16] studied the two-body problem with varying mass in case of isotropic mass loss. In that work, the problem was treated analytically in the Hamiltonian frame-work and the equations of motion ware integrated using the Lie operator and Lie series.

Our work is aimed to introduce an analytical solution for the problem of two bodies with varying mass, taking into consideration the periastron effects, using the canonical perturbation technique derived by Hori and developed by Kamel.

\section{The Hamiltonian of the Problem}

The Hamiltonian is constructed in terms of Delaunay's variables as described in Deprit [17]:

$$
\mathscr{K}^{*}(\ell, L, G ; t)=-\frac{1}{2} \frac{\mu^{2}}{L^{2}}+\frac{\dot{\mu}}{\mu} L e \sin E
$$

The first term in the Hamiltonian function, described by Equation (3), is the Hamiltonian in the Keplerian case (with constant mass) $\mu_{0}$ while the second term represents the varying mass effects. In terms of the Delaunay variable, we can develop the Hamiltonian as:

$$
\mathscr{K}^{*}=-\frac{\mu_{o}^{2}}{2 L_{1}^{2}}+\frac{\dot{\mu}}{\mu}\left(L_{1}^{2}-L_{2}^{2}\right)^{\frac{1}{2}} \sin E
$$

That is meant that the Hamiltonian is function of $\ell_{1}, L_{1}$ and $L_{2}$ as:

$$
\mathscr{K}^{*}=\mathscr{K}^{*}\left(\ell_{1}, \ldots, \ldots, L_{1}, L_{2}, \ldots,-\right)
$$

The variation of $\mu$ may be retained from one of the two masses $m_{1}$ or $m_{2}$ and this is the case of one body 
eject mass. Otherwise the case of the two bodies ejects masses. We will concern with the first case. Then;

$$
\dot{\mu} \equiv \dot{m}_{1}
$$

The Hamiltonian $\mathscr{K}^{*}$ represented by Equation (4) is implicit depending on time through the variable mass $\mu$ and its time derivative $\dot{\mu}$. By modifying Docobo's law for the rate of change of mass assigned by Equation (2) and use Jeans law described by Equation (1) we get:

$$
\dot{\mu}\left(r, t, L_{2}\right)=-\alpha m^{n}-\beta \frac{L_{2} \mu^{2}}{L_{1}^{4}}\left(\frac{a}{r}\right)^{2}
$$

By applying Equation (6) in the Hamiltonian function (4) which can be written as:

$$
\mathscr{K}^{*}=\frac{-\mu_{o}^{2}}{2 L_{1}^{2}}-\alpha \mu^{n-1}\left(L_{1}^{2}-L_{2}^{2}\right)^{\frac{1}{2}} \sin E-\beta L_{2} \frac{\mu}{L_{1}^{4}}\left(L_{1}^{2}-L_{2}^{2}\right)^{\frac{1}{2}}\left(\frac{a}{r}\right)^{2} \sin E
$$

with $\boldsymbol{n}$ positive number varying between $\mathbf{1 . 4}$ and 4.4.

Since the variable mass can be expressed as a Taylor series expansion as:

$$
\mu=\mu_{0}+\left.\frac{\mathrm{d} \mu}{\mathrm{d} t}\right|_{t=t_{0}} \frac{\left(t-t_{0}\right)}{1 !}+\left.\frac{\mathrm{d}^{2} \mu}{\mathrm{d} t^{2}}\right|_{t=t_{0}} \frac{\left(t-t_{0}\right)^{2}}{2 !}+\cdots
$$

that will lead to a Hamiltonian with explicit dependence of time. So we must extend the phase space by introducing a new pair of variable $\left(\ell_{4}, L_{4}\right)$. The first is " $\ell_{4}$ " assigned as the variable mass and the second its conjugate momentum " $L_{4}$ ”.

The new systems of canonical equations of motion are:

$$
\dot{\ell}_{i}=\frac{\partial K}{\partial L_{i}} \text { and } \dot{L}_{i}=-\frac{\partial K}{\partial \ell_{i}} \quad i=1, \cdots, 4
$$

where $K$ is the new Hamiltonian in the extended phase space which can be expressed as:

$$
K=\frac{-\mu_{o}^{2}}{2 L_{1}^{2}}-\alpha \ell_{4}^{n} L_{4}-\alpha \mu^{n-1}\left(L_{1}^{2}-L_{2}^{2}\right)^{\frac{1}{2}} \sin E-\beta L_{2} \frac{\mu}{L_{1}^{4}}\left(\frac{a}{r}\right)^{2}\left(L_{1}^{2}-L_{2}^{2}\right)^{\frac{1}{2}} \sin E
$$

The first term in Equation (9) is contribution of the two body with constant mass with $\mu_{o}$ is total mass of the system at specific time $t_{0}$. The small parameter $\beta$ is close to zero as well as the small parameter $\alpha$. In this stage, we can handle the problem as two bodies with two small parameters. But, if we assume that the two small parameters $\alpha$ and $\beta$ has the same order of magnitude the problem will turn into one small parameter problem. The Hamiltonian (9) can be written in the form:

$$
K=\sum_{i=0}^{1} \alpha^{i} K_{i}
$$

where,

$$
K_{o}=-\frac{\mu_{o}}{2 L_{1}^{2}}
$$

with,

$$
K_{1}=\sum_{i=1}^{2} K_{1 i}
$$

and,

$$
\begin{aligned}
& K_{11}=-L_{4} \ell_{4}^{n}-\left(L_{1}^{2}-L_{2}^{2}\right)^{\frac{1}{2}} \ell_{4}^{n-1} \sin E \\
& K_{12}=-\frac{\beta}{\alpha} \frac{L_{2}}{L_{1}^{4}}\left(L_{1}^{2}-L_{2}^{2}\right)^{\frac{1}{2}} \ell_{4}\left(\frac{a}{r}\right)^{2} \sin E .
\end{aligned}
$$

The canonical equations of motion for the Hamiltonian $K$ are:

$$
\dot{\ell}_{i}=\frac{\partial K}{\partial L_{i}} \text { and } \dot{L}_{i}=-\frac{\partial K}{\partial \ell_{i}} \quad i=1, \cdots, 4
$$


Applying the last Equation for the Hamiltonian function represented by Equation (10) yields:

$$
\begin{aligned}
& \dot{\ell}_{1}=\frac{\mu_{o}^{2}}{L_{1}^{3}}-\alpha\left(\ell_{4}^{n-1} \frac{L_{1}^{2}}{e} \sin E\right)+\frac{\beta L_{2}}{\ell_{4} r^{2} e}\left(\left(1-4 e^{2}\right) \sin E+\frac{2}{r}\left(\frac{L_{2}}{L_{1}}\right)^{2} \cos E\right) \\
& \dot{\ell}_{2}=-\alpha\left(\ell_{4}^{n-1}\left(\frac{L_{2}}{L_{1} e}\right) \sin E\right)+\frac{\beta}{\ell_{4} L_{1} r^{2}} \sin E\left[L_{1}^{2} e-\frac{L_{2}^{2}}{e}-\frac{2 L_{2}^{2} a}{r} \cos E\right] . \\
& \dot{\ell}_{3}=\frac{\partial K}{\partial L_{3}}=0 \\
& \dot{\ell}_{4}=\frac{\partial K}{\partial L_{4}}=-\alpha \ell_{4}^{n} \\
& \dot{L}_{1}=-\alpha\left(\ell_{4}^{n-1} L_{1} \frac{e a}{r}\right)+\beta \frac{L_{2} L_{1} a e}{\ell_{4} r^{3}} \cdot\left(\cos E-2 \frac{e}{r} \sin ^{2} E\right) \\
& \dot{L}_{2}=\frac{\partial K}{\partial \ell_{2}}=0 \\
& \dot{L}_{3}=\frac{\partial K}{\partial \ell_{3}}=0 \\
& \dot{L}_{4}=\frac{\partial K}{\partial \ell_{4}}=-\alpha\left[L_{4} n \ell_{4}^{n-1}+L_{1} e \sin E(n-1) \ell_{4}^{n-2}\right]+\beta \frac{L_{2}}{L_{1}^{3}} e\left(\frac{a}{r}\right)^{2} \sin E
\end{aligned}
$$

\section{Short Period Solution Using Hori Method's}

The Hameltonian function $K$ is function of $\ell_{1}, \ell_{4}, L_{1}, L_{2}$, and $L_{4}$. The integrable part of the Hameltonian, $K_{0}$, is function of $L_{1}$. So the variable $\ell_{1}$ can be consedered as the fast variable.

In the next stage we will use Hori's method [18], developed by Kamel [19] to elemeinate the short period termes from the Hameltonian. And the transformed Hameltonian, $K^{*}$, can be written, up to second orders, as follows:-

\subsection{Zero Order}

By applying the Hori’s Perturbation technique developed by Kamel yields:

$$
K_{o}^{*}=K_{o}\left(\_; L_{1}^{\prime}\right)=\frac{-\mu_{o}^{2}}{2 L_{1}^{\prime 2}}
$$

\subsection{First Order}

The first order transformed Hamiltonian derived by Horis' can be outlined as:-

where;

$$
K_{1}^{*}=\tilde{K}_{1}+\left(K_{o} ; S_{1}\right)
$$

And by choosing;

$$
\tilde{K}_{1}=K_{1}
$$

$$
K_{1}^{*}=\left\langle\tilde{K}_{1}\right\rangle_{\ell_{1}}
$$

then

$$
K_{1 p}^{*}=\tilde{K}_{1}-K_{1}^{*}=\left(S_{1} ; K_{o}\right)
$$

So the first order generating function, $S_{1}$, is therefore obtained by integral: 


$$
S_{1}=\left(\frac{\partial K_{o}}{\partial L_{1}}\right)^{-1} \int K_{1 p}^{*} \partial \ell_{1}
$$

By averaging the Hameltonian, $K_{1}$, over the mean anomaly $\ell_{1}$ we can determine the transformed first order Hamiltonian, $K_{1}^{*}$, as:-

$$
K_{1}^{*}=\frac{1}{2 \pi} \int_{0}^{2 \pi}\left[\left(-\ell_{4}^{n} L_{4}-\ell_{4}^{n-1} L_{1} e \sin E\right)-\frac{\beta}{\alpha} \frac{L_{2}}{L_{1}^{3}} e \ell_{4}\left(\frac{a}{r}\right)^{2} \sin E\right] \partial \ell_{1} .
$$

After calculating the required integrals needed in the last equation we get:

$$
K_{1}^{*}=-\ell_{4}^{n} L_{4} .
$$

By applying Equation (13.4) to calculate the periodic part of the Hamiltonian as:

$$
K_{1 p}^{*}=\tilde{K}_{1}-K_{1}^{*}=K_{1}-K_{1}^{*}=-\ell_{4}^{n-1} L_{1} e \times \sin E-\frac{\beta}{\alpha} \frac{L_{2}}{L_{1}^{3}} e \ell_{4}\left(\frac{a}{r}\right)^{2} \sin E
$$

To calculate the first order generating function, $S_{1}$, we must use Equation (13.5) (with the help of Equations (12) and (15)) which lead, after calculate the required derevativs and products, to the results:-

$$
\therefore S_{1}=\sum_{i=1}^{2} B_{i}^{c} \cos i E+B_{o} \ln \left(\frac{r}{a}\right)
$$

where $B$ 's are function of $\left(\ell_{4} ; L_{1}, L_{2}\right)$ and given by:

$$
\begin{gathered}
B_{o}=-\frac{\beta}{\alpha} \frac{L_{2}}{\mu_{o}^{2}} \ell_{4} \\
B_{1}^{c}=\frac{L_{1}^{4}}{\mu_{o}^{2}} e \ell_{4}^{n-1} \\
B_{2}^{c}=\frac{-L_{1}^{4}}{4 \mu_{o}^{2}} e^{2} \ell_{4}^{n-1}
\end{gathered}
$$

\subsection{Second Order}

The seconed order transformed Hamiltonian derived by Horis’ can be outlined as:-

$$
K_{2}^{*}=\tilde{K}_{2}+\left(K_{o} ; S_{2}\right) \text {, }
$$

where;

$$
\tilde{K}_{2}=K_{2}+\left(K_{1}+K_{1}^{*} ; S_{1}\right) .
$$

Choosing;

$$
K_{2}^{*}=\left\langle\tilde{K}_{2}\right\rangle_{\ell_{1}},
$$

and;

$$
K_{2 p}^{*}=\tilde{K}_{2}-K_{2}^{*}=\left(S_{1} ; K_{o}\right) .
$$

Hence $S_{2}$ therefore can be obtained by;

$$
S_{2}=\left(\frac{\partial K_{o}}{\partial L_{1}}\right)^{-1} \int K_{2 p}^{*} \mathrm{~d} \ell_{1}
$$

To construct the function $\tilde{K}_{2}$, we need, first, to calculate the Poisson bracket $\left(K_{1}+K_{1}^{*} ; S_{1}\right)$. By the help of 
Equations (10.2), (14) and (16) with $K_{2}=0$ yeilds:

$$
\tilde{K}_{2}=\sum_{g=2}^{5} \sum_{f=-1}^{4} C_{g, f}^{c}\left(\frac{a}{r}\right)^{g} \operatorname{Cos} f E-\sum_{t=2}^{6} \sum_{s=-1}^{4} C_{t, s}^{c}\left(\frac{a}{r}\right)^{t} \operatorname{Cos} E+2 \ell_{4}^{n} \sum_{i=1}^{2} B_{i}^{* c} \operatorname{CosiE}+2 \ell_{4}^{n} B_{0}^{*} \ln \left(\frac{r}{a}\right)
$$

where the coeffecients $C_{g, f}^{c}, C_{t, s}^{c}$ and $B_{i}^{* c}$ are function of $\left(\ell_{4} ; L_{1}, L_{2}\right)$ defined as:-

$$
\begin{aligned}
& C_{g, f}^{c}=\sum_{m=1}^{2} \sum_{u=0}^{2}\left(\frac{1}{2}\right) A_{m, u}^{c}\left[B_{g-2 m, u-f}^{c}+B_{g-2 m, f-u}^{c}\right] . \\
& C_{t, s}^{c}=\sum_{m^{\prime}=0}^{2} \sum_{u^{\prime}=1}^{2}\left(\frac{1}{2}\right) A_{m^{\prime}, u^{\prime}}^{s i}\left[B_{\frac{1}{2} t\left(-\left(m^{\prime}+1\right), u^{\prime}-s\right.}^{s i}-B_{\frac{1}{2} t-\left(m^{\prime}+1\right), u^{\prime}-s}^{s i}\right] . \\
& B_{i}^{* c}=\frac{\partial B_{i}^{c}}{\partial \ell_{4}}
\end{aligned}
$$

Then, applying Equation (17.2) by averaging over the mean anomaly $\ell_{1}$ for the last Equation (18), we can determine the transformed second order Hamiltonian, $K_{2}^{*}$, as:-

$$
K_{2}^{*}=\left\langle\tilde{K}_{2}\right\rangle_{\ell_{1}}=\left\langle\left(K_{1}+K_{1}^{*} ; S_{1}\right)\right\rangle_{\ell_{1}}=\frac{1}{2 \pi} \int_{0}^{2 \pi}\left(K_{1}+K_{1}^{*} ; S_{1}\right) \partial \ell_{1} .
$$

Substituting from Equation (18) in the last Equation yeilds:-

$$
\begin{aligned}
K_{2}^{*}=\frac{1}{2 \pi}\left\{\int_{0}^{2 \pi}\left[\sum_{g=2}^{5} \sum_{f=-1}^{4} C_{g, f}^{c}\left(\frac{a}{r}\right)^{g} \cos f E\right] \partial \ell_{1}-\int_{0}^{2 \pi}\left[\sum_{t=2}^{6} \sum_{s=-1}^{4} C_{t, s}^{c}\left(\frac{a}{r}\right)^{t} \cdot \cos E\right] \partial \ell_{1}\right. \\
\left.+\int_{0}^{2 \pi} 2 \ell_{4}^{n}\left[\sum_{i=1}^{2} B_{i}^{* c} \cos i E\right] \partial \ell_{1}+2 \ell_{4}^{n} \int_{0}^{2 \pi} B_{0}^{*} \ln \left(\frac{r}{a}\right) \partial \ell_{1}\right\}
\end{aligned}
$$

A useful paper for Ahmed (1994) [20], was used to calculate the required integral. While the last integral was solved except for the case of hyperbolic orbits.

$$
K_{2}^{*}=\ell_{4}^{n}\left\{e B_{1}^{*_{c}}-2 B_{o}^{*}\left(-1+\sqrt{1-e^{2}}+\ln [2]-\ln \left[1+\sqrt{1-e^{2}}\right]\right)\right\}
$$

where $B_{o}^{*}$ and $B_{1}^{*_{c}}$ are coefficients, ware derived during the calculation of the Poisson brackets $\left(K_{1}+K_{1}^{*} ; S_{1}\right)$, defined as:

$$
\begin{aligned}
& B_{o}^{*}=\frac{\partial}{\partial \ell_{4}}\left(B_{o}\right)=-\frac{\beta}{\alpha} \frac{L_{2}}{\mu_{o}^{2}} . \\
& B_{1}^{*_{c}}=\frac{\partial}{\partial \ell_{4}}\left(B_{1}^{c}\right)=\frac{e L_{1}^{4}}{\mu_{o}^{2}}(n-1) \ell_{4}^{n-2} .
\end{aligned}
$$

Finally, the required transformed second order Hamiltonian can be written as:-

$$
K_{2}^{*}=\frac{1}{\mu_{o}^{2}}\left[e^{2} L_{1}^{4}(n-1) \ell_{4}^{2 n-2}+2 \ell_{4}^{n} \frac{\beta}{\alpha} \Psi\right]
$$

where $\Psi$ is function of $L_{1}$ and $L_{2}$ and defined by:

$$
\Psi=L_{2}\left[-0.3068528194+\sqrt{1-e^{2}}-\ln \left(1+\sqrt{1-e^{2}}\right)\right]
$$

The last, second orders Hamiltonian, is valid in all cases of orbits except in the case of hyperbolic orbits ( $e>$ 1).

\subsection{The Transformed Hamiltonian}

The second order transformed Hamiltonian; $K^{*}$ can be constructed in the form:

$$
K^{*}=K_{o}^{*}+\alpha K_{1}^{*}+\alpha^{2} K_{2}^{*} .
$$


Which can be written by using Equations (12), (14) and (21) as:

$$
K^{*}=\frac{-\mu_{o}^{2}}{2 L_{1}^{2}}+\alpha\left[-\ell_{4}^{n} L_{4}\right]+\frac{\alpha^{2}}{2 \mu_{o}^{2}}\left[e^{2} L_{1}^{4}(n-1) \ell_{4}^{2 n-2}+2 \ell_{4}^{n} \frac{\beta}{\alpha} \Psi\right] .
$$

The equations of motion in the transformed augmented phase space can be derived as follows:

$$
\begin{aligned}
& \dot{\ell}_{1}=\frac{\mu_{0}^{2}}{L_{1}^{3}}+\frac{\alpha^{2}}{2 \mu_{0}^{2}}\left[\left(4 L_{1}^{3}+2 L_{1} L_{2}^{2}\right)(n-1) \ell_{4}^{2 n-2}-2 \ell_{4}^{n} \frac{\beta}{\alpha}\left(\frac{L_{2}}{L_{1}}\right)^{3}\left(\frac{1}{1+\sqrt{1-e^{2}}}\right)\right] \\
& \dot{\ell}_{2}=\frac{\alpha^{2}}{2 \mu_{o}^{2}}\left[-2 L_{1}^{4}(n-1) \ell_{4}^{2 n-2} \frac{L_{2}}{L_{1}^{2}}+2 \ell_{4}^{n} \frac{\beta}{\alpha} \Psi_{; L_{2}}\right] . \\
& \dot{\ell}_{3}=0 . \\
& \dot{\ell}_{4}=-\alpha \ell_{4}^{n} . \\
& \dot{L}_{1}=0 . \\
& \dot{L}_{2}=0 . \\
& \dot{L}_{3}=0 . \\
& \dot{L}_{4}=\alpha n \ell_{4}^{n-1} L_{4}-\frac{\alpha^{2}}{\mu_{0}^{2}}\left[L_{1}^{4} e^{2}(n-1)(2 n-2) \ell_{4}^{2 n-3}+2 n \frac{\beta}{\alpha} \ell_{4}^{n-1} \Psi\right]
\end{aligned}
$$

where $\Psi_{; L_{2}}$ is the partial differentiation for the function $\Psi$ with respect to the momentum $L_{2}$ and given by:

$$
\Psi_{; L_{2}}=\frac{1}{L_{1}^{2}\left(1+\sqrt{1-e^{2}}\right)}\left\{2 L_{2}^{2}+L_{1}^{2} \cdot\left[-0.3068528194+0.693147 \sqrt{1-e^{2}}+\left(1+\sqrt{1-e^{2}}\right) \ln \left(1+\sqrt{1-e^{2}}\right)\right]\right\}
$$

The Hamiltonian and the system of differential equation, described by Equations (23), has one degree of freedom since the variable $\ell_{4}$ is explicitly appears in them.

\subsection{The Element of Short Period Transformation and Its Inverse}

The elements of short period transformation can be calculated using equations:

$$
\begin{aligned}
& \ell_{i}=\ell_{i}^{\prime}+\alpha \frac{\partial}{\partial L_{i}^{\prime}} S_{1}+O\left(\alpha^{2}\right) . \\
& L_{i}=L_{i}^{\prime}+\alpha \frac{\partial}{\partial \ell_{i}^{\prime}} S_{1}+O\left(\alpha^{2}\right) .
\end{aligned}
$$

The inverse transformation equations can be calculated as follows:

$$
\begin{gathered}
\ell_{i}^{\prime}=\ell_{i}-\alpha \frac{\partial}{\partial L_{i}^{\prime}} S_{1}-O\left(\alpha^{2}\right) . \\
L_{i}^{\prime}=L_{i}+\alpha \frac{\partial}{\partial \ell_{i}^{\prime}} S_{1}+O\left(\alpha^{2}\right) .
\end{gathered}
$$

where $\frac{\partial}{\partial L_{1}^{\prime}} S_{1}, \frac{\partial}{\partial \ell_{1}^{\prime}} S_{1}, \frac{\partial}{\partial \ell_{4}^{\prime}} S_{1}$ and $\frac{\partial}{\partial L_{2}^{\prime}} S_{1}$ can be calculated using Equation (13.5).

The prime over the variables means that variables in the transformed phase space which will be omitted for the sake of simplicity. 
The variable mass $\ell_{4}$ is implicitly function of time through the relation (7'), so we will complete the solution using the method which was introduced by Delva [14] and Hanslmeier [15], separately.

\section{The Final Solution}

A special linear differential operator, the Lie operator, produces a Lie series to construct an approximate solution. The convergence of the Lie series is similar to that of Taylor series, since the Lie is only another analytic form of the Taylor series. The algorithm for the method was outlined in Delva [14] and Hanslmeier [15].

The Lie operator, $D$, can be defined as:

$$
D X\left(Q_{j}, P_{j} ; t\right)=\frac{\mathrm{d}}{\mathrm{d} t} X\left(Q_{j}, P_{j} ; t\right)=\sum_{j=1}^{4}\left(\frac{\partial K^{*}}{\partial L_{j}} \frac{\partial}{\partial \ell_{j}}-\frac{\partial K^{*}}{\partial \ell_{j}} \frac{\partial}{\partial L_{j}}\right)+\frac{\partial}{\partial t}
$$

and the second order " $D$ " operator, which can be noted as $D^{2}$, constructed as:

$$
D^{2} X=\frac{\mathrm{d}}{\mathrm{d} t} D X
$$

The coefficients of the operator $D, \frac{\partial K^{*}}{\partial \ell_{j}}$ and $\frac{\partial K^{*}}{\partial L_{j}}$, can be replaced by the canonical equations of motion represented by Equations (23). Then, Lie operator for our case can be written as:

$$
\begin{aligned}
D= & \left\{\frac{\mu_{0}^{2}}{L_{1}^{3}}+\frac{\alpha^{2}}{2 \mu_{0}^{2}}\left[\left(4 L_{1}^{3}+2 L_{1} L_{2}^{2}\right)(n-1) \ell_{4}^{2 n-2}-2 \ell_{4}^{n} \frac{\beta}{\alpha}\left(\frac{L_{2}}{L_{1}}\right)^{3}\left(\frac{1}{1+\sqrt{1-e^{2}}}\right)\right]\right\} \frac{\partial}{\partial \ell_{1}} \\
& +\frac{\alpha^{2}}{2 \mu_{o}^{2}}\left[-2 L_{1}^{4}(n-1) \ell_{4}^{2 n-2} \frac{L_{2}}{L_{1}^{2}}+2 \ell_{4}^{n} \frac{\beta}{\alpha} \Psi_{; L_{2}}\right] \frac{\partial}{\partial \ell_{2}} \\
& -\alpha \ell_{4}^{n} \frac{\partial}{\partial \ell_{4}}+\left\{\alpha n \ell_{4}^{n-1} L_{4}-\frac{\alpha^{2}}{\mu_{0}^{2}}\left[L_{1}^{4} e^{2}(n-1) \cdot(2 n-2) \ell_{4}^{2 n-3}+2 n \frac{\beta}{\alpha} \ell_{4}^{n-1} \Psi\right]\right\} \cdot \frac{\partial}{\partial L_{4}}+\frac{\partial}{\partial t} .
\end{aligned}
$$

Now, we can calculate the solution by applying the $D$ operator, represented by Equation (27), up to second orders, to the variables $\ell_{i}, L_{i}$ and the results are as follows:

$$
\begin{aligned}
\ell_{1}= & \ell_{1, o} \\
+ & \left.+\frac{\mu_{0}^{2}}{L_{1}^{3}}+\frac{\alpha^{2}}{2 \mu_{0}^{2}}\left[\left(4 e^{2} L_{1}^{3}+2 L_{1} L_{2}^{2}\right) \cdot(n-1) \ell_{4}^{2 n-2}+\frac{2 \ell_{4}^{n}}{\sqrt{1-e^{2}}} \frac{\beta}{\alpha}\left(\frac{L_{2}}{L_{1}}\right)^{3} \cdot\left(-1+\frac{1}{1+\sqrt{1-e^{2}}}\right)\right]\right\}\left.\right|_{\ell_{1}=\ell_{1, o}} \cdot \frac{\left(t-t_{o}\right)}{1 !} \\
& -\left.\frac{\alpha^{3}}{2 \mu_{0}^{2}}\left[\left(4 e^{2} L_{1}^{3}+2 L_{1} L_{2}^{2}\right)(n-1)(2 n-2) \cdot \ell_{4}^{2 n-3}+\frac{2 n \ell_{4}^{n-1}}{\sqrt{1-e^{2}}} \frac{\beta}{\alpha}\left(\frac{L_{2}}{L_{1}}\right)^{3} \cdot\left(-1+\frac{1}{1+\sqrt{1-e^{2}}}\right)\right]\right|_{\ell_{1}=\ell_{1, o}} \frac{\left(t-t_{o}\right)^{2}}{2 !}+\cdots \\
\ell_{2}= & \ell_{2, o} \\
& +\left.\frac{\alpha^{2}}{2 \mu_{o}^{2}}\left[2 \frac{\beta}{\alpha} \frac{L_{2}^{2}}{L_{1}^{2}} \frac{\ell_{4}^{n}}{\sqrt{1-e^{2}}} \cdot\left(1-\frac{1}{1+\sqrt{1-e^{2}}}\right)-2 L_{1}^{2} L_{2}(n-1) \ell_{4}^{2 n-2}+2 \frac{\ell_{4}^{n}}{L_{2}} \frac{\beta}{\alpha} \Psi\right]\right|_{\ell_{2}=\ell_{2, o}} \frac{\left(t-t_{o}\right)}{1 !} \\
& -\left.\frac{\alpha^{3} \ell_{4}}{2 \mu_{o}^{2}}\left[2 \frac{\beta}{\alpha} \frac{L_{2}^{2}}{L_{1}^{2}} \frac{\ell_{4}^{n-1}}{\sqrt{1-e^{2}}}\left(1-\frac{1}{1+\sqrt{1-e^{2}}}\right)-2 L_{1}^{2} L_{2}(n-1)(2 n-2) \ell_{4}^{2 n-3}+2 n \frac{\ell_{4}^{n-1}}{L_{2}} \cdot \frac{\beta}{\alpha} \Psi\right]\right|_{\ell_{2}=\ell_{2, o}} \frac{\left(t-t_{o}\right)^{2}}{2 !}+\cdots \\
\ell_{3}= & \ell_{3, o} \\
\ell_{4}= & \ell_{4, o}-\left.\alpha \ell_{4}^{n}\right|_{\ell_{4}=\ell_{4, o}} \cdot \frac{\left(t-t_{o}\right)}{1 !}+\left.n \alpha^{2} \ell_{4}^{2 n-1}\right|_{\ell_{4}=\ell_{4, o}} \cdot \frac{\left(t-t_{o}\right)^{2}}{2 !}+\cdots
\end{aligned}
$$




$$
\begin{aligned}
L_{1}= & L_{1, o} \\
L_{2}= & L_{2, o} \\
L_{3}= & L_{3, o} \\
L_{4}= & L_{4, o}+\left.\left\{\alpha n \ell_{4}^{n-1} L_{4}-\frac{\alpha^{2}}{2 \mu_{0}^{2}}\left[L_{1}^{4} e^{2}(n-1) \cdot(2 n-2) \ell_{4}^{2 n-3}+2 \frac{\beta}{\alpha} n \ell_{4}^{n-1} \Psi\right]\right\}\right|_{L_{4}=L_{4, o}} \\
& \cdot \frac{\left(t-t_{o}\right)}{1 !}+\left.\left\{\alpha^{2} \ell_{4}^{2 n-2} L_{4} n-\frac{\alpha^{3}}{2 \mu_{0}^{2}} \cdot\left[2 \frac{\beta}{\alpha} n \ell_{4}^{2 n-2} \Psi-\alpha L_{1}^{4} e^{2}(n-1)(2 n-3) \cdot(2 n-2) \ell_{4}^{3 n-4}\right]\right\}\right|_{L_{4}=L_{4, o}} \frac{\left(t-t_{o}\right)^{2}}{2 !}+\cdots
\end{aligned}
$$

where the quantities $\ell_{1, o}, \ell_{2, o}, \ell_{3, o}, \ell_{4, o}, L_{1, o}, L_{2, o}, L_{3,0}$ and $L_{4,0}$ are the orbital elements at time $t_{0}$ Using the initial values for the orbital elements, with the help of Equations (28) - (35), we can calculate the new perturbed orbital elements due to the varying mass.

\section{Discussion and Conclusions}

The last group of equations is a second order solution for the two body problem with $\boldsymbol{m}_{\mathbf{1}}$ varying mass. In those equations, the following results are included.

1 . The varying mass gives rise to the change of mean anomaly $\ell_{1}$ and the argument of preside $\ell_{2}$. That rises influenced by both the varying mass and the periastron effect.

2. A new varying, second order, mass formula was derived and represented by Equation (31).

3. The varying mass, as well as the periastron effects, does not affect the argument of ascending node $\ell_{3}$ and the momenta $L_{1}, L_{2}$ and $L_{3}$ in the extended transformed phase space.

4. A second order equation for calculation of the momentum associated to the varying mass, $L_{4}$, as well as the periastron effect, was derived and represented by Equation (35).

Future work: We plane to apply these equations for deferent systems as well as comparing the results with others and with observations.

\section{References}

[1] Polyakhova, E.N. (1994) A Two-Body Variable-Mass Problem in Celestial Mechanics: The Current State. Astronomy Reports, 38, 283-291.

[2] Prieto, C. (1995) Publicaciones del Departamento de Matematica Aplicada, Universidade de Santiago de CompostelaSpain.

[3] Hadjidemetriou, J. (1963) Two-Body Problem with Variable Mass: A New Approach. Icarus, 2, 440-451. http://dx.doi.org/10.1016/0019-1035(63)90072-1

[4] Hadjidemetriou, J. (1966) Analytic Solutions of the Two-Body Problem with Variable Mass. Icarus, 5, 34-46. http://dx.doi.org/10.1016/0019-1035(66)90006-6

[5] Jeans, J.H. (1924) Cosmogonic Problems Associated with a Secular Decrease of Mass. MNRAS, 85, 2-11.

[6] Jeans, J.H. (1925) The Effect of Varying Mass on a Binary System. MNRAS, 85, 912-925.

[7] Prieto C. and Docobo, J.A. (1997) Analytic Solution of the Two-Body Problem with Slowly Decreasing Mass. Astronomy \& Astrophysics, 318, 657-661.

[8] Prieto, C. and Docobo, J.A. (1997) On the Two-Body Problem with Slowly Decreasing Mass. Celestial Mechanics and Dynamical Astronomy, 68, 53-62. http://dx.doi.org/10.1023/A:1008235630740

[9] Docobo, J.A., Blanco, J. and Abelleira, P. (1999) Monografias de la Academiade Ciencias de Zaragoza, 14, II Jornadas de Mecanica Celeste, Academia de Ciencias de Zaragoza, Zaragoza, 33. (in Spanish)

[10] Andrade, M. and Docobo, J.A. (2000) The Influence of Decreasing Mass on the Orbits of Wide Binaries: An Approach to the Problem. Proceedings of the 4th Scientific Meeting of the Spanish Astronomical Society (SEA), Santiago de Compostela, 11-14 September 2000, 273.

[11] Andrade, M. and Docobo, J.A. (2002) The Influence of Mass Loss on Orbital Elements of Binary Systems by Periastron Effect. International Conference on Classical Nova Explosions, AIP Conference Proceedings, Santiago de Compostela, 82-85. 
[12] Andrade, M. and Docobo, J.A. (2003) Orbital Dynamics Analysis of Binary Systems in Mass-Loss Scenarios. Conference to Honor John Dyson, Pátzcuaro, Michoacán, México, Santiago de Compostela, 223-225.

[13] Rahoma, W.A., et al. (2009) Analytical Treatment of the Two-Body Problem with Slowly Varying Mass. Journal of Astrophysics and Astronomy, 30, 187-205. http://dx.doi.org/10.1007/s12036-009-0012-y

[14] Delva, M. (1984) Integration of the Elliptic Restricted Three-Body Problem with Lie Series. Celestial Mechanics, 34, 145-154. http://dx.doi.org/10.1007/BF01235797

[15] Hanslmeier, A. (1984) Application of Lie-Series to Regularized Problems in Celestial Mechanics. Celestial Mechanics, 34, 135-143. http://dx.doi.org/10.1007/BF01235796

[16] Rahoma, W.A., et al. (2011) Two-Body Problem with Varying Mass in Case of Isotropic Mass Loss. Advances in Theoretical and Applied Mechanics, 4, 69-80.

[17] Deprit, A. (1983) The Secular Accelerations in Gylden’s Problem. Celestial Mechanics, 31, 1-22. http://dx.doi.org/10.1007/BF01272557

[18] Hori, G. (1966) Theory of General Perturbation with Unspecified Canonical Variable. Astronomical Society of Japan, 18, 287.

[19] Kamel, A.A. (1969) Expansion Formulae in Canonical Transformations Depending on a Small Parameter. Celestial Mechanics, 1, 190-199. http://dx.doi.org/10.1007/BF01228838

[20] Ahmed, M.K.M. (1994) On the Normalization of Perturbed Keplerian Systems. The Astronomical Journal, 107, 19001903. http://dx.doi.org/10.1086/117001 